\title{
Hepatopancreatoduodenectomy-lesson's with 35 patients over 10 years
}

\begin{abstract}
Hepatopancreatoduodenectomy (HPD) represents the most complex abdominal operation comprising generally large resections of hepatic parenchyma combined with gastro-duodenum-pancreatectomy, in which its main indications include biliary tract neoplasia. Surgical resection is considered the only potential modality of cure in the treatment of malignant biliary tumors, including gallbladder cancer and cholangiocarcinomas. High morbidity and mortality rates still affect a large part of the patients submitted to this operation due to extensive resections of hepatic parenchyma associated with critical anastomoses of bile ducts and pancreatic gland.
\end{abstract}

In Brazil, there is only one relevant publication about HPD in biliary cancer and some isolated groups in Rio Grande do Sul, São Paulo, Maranhão and Rio de Janeiro have gained experience in this operation. In three different institutions in Rio de Janeiro, the Hospital Adventista Silvestre, Hospital São Lucas and Hospital Universitario Clementino Fraga Filho-UFRJ, 35 patients underwent HPD between 2004 and 2014, representing, in our knowledge, the largest worldwide experience outside of Japan. We report a multicenter retrospective study

Our group has pioneered the radical operations of biliary carcinoma with HPD in Brazil. However, the initial results were poor with high rates of pancreatic fistulas and hepatic failure with subsequent sepsis and death. The use of protocols from the accumulated experience of the Japanese school, such as the oral replacement of bile in jaundiced patients, routine portal embolization, immunonutrition and the greater use of centralectomies in gallbladder carcinomas instead of trisectionectomy have shown a significant improvement in the results. In the 35 cases evaluated, the overall mortality was $34.2 \%$ and in 17 patients $(53 \%)$ vascular resections and reconstruction were applied.

HPD is a complex abdominal operation with a high mortality rate and a long learning curve. We emphasize that this operation should be limited to specialized centers with experience in liver transplantation background and advanced hepatobiliopancreatic surgery. Aggressive preoperative management with biliary drainage and portal vein embolization are crucial maneuvers to obtain satisfactory results. The HPDs for gallbladder cancer is feasible but requires a careful patient selection.

Keywords: hepatopancreatoduodenectomy, gallbladder cancer, radical surgery, klatskin, cholangiocarcinomas
Volume 3 Issue 6 - 2017

\author{
Eduardo de Souza Martins Fernandes, ${ }^{1,2}$ \\ Caio Márcio Vieira de Oliveira, ${ }^{2}$ Munique \\ Siqueira, ${ }^{2}$ Felipe Mello, ${ }^{2}$ Ronaldo Andrade, ${ }^{2}$ \\ Camila Girão, ${ }^{2}$ Leandro Pimentel, ${ }^{2}$ Antonio \\ Augusto Sousa' \\ 'Department of Surgery, Rio de Janeiro Federal University, Brazil \\ ${ }^{2}$ Department of Surgery and Transplantation, Rio de Janeiro \\ Adventist Hospital, Brazil
}

\begin{abstract}
Correspondence: Eduardo de Souza Martins Fernandes, Department of Surgery, Rio de Janeiro Federal University, Brazil, Email esmfernandes@hotmail.com
\end{abstract}

Received:September 19,2017 | Published: November 29, 2017

\section{Abbrevations: $\quad$ PD: Pancreatoduodenectomy;} HLPD: Hepatoligamentopacreatoduodenectomy; HPD: Hepatopancreatoduodenectomy

\section{Aim}

Hepatopancreatoduodenectomy (HPD) is the most complex abdominal surgery and performed in only a few groups in Brazil. Gallbladder cancer remains a reserved prognosis disease and in selected cases, radical resection (R0) surgery allows survival even in advanced tumors. Cholangiocarcinomas represent the main indication for HPD with promising results.

\section{Introduction}

Hepatopancreatoduodenectomy(HPD) represents themostcomplex abdominal operation comprising generally large resections of hepatic parenchyma combined with gastro-duodenum-pancreatectomy, in which its main indications include biliary tract neoplasia. Surgical resection is considered the only potential modality of cure in the treatment of malignant biliary tumors, including gallbladder cancer and cholangiocarcinomas. High morbidity and mortality rates still affect a large part of the patients submitted to this operation due to extensive resections of hepatic parenchyma associated with critical anastomoses of bile ducts and pancreatic gland. ${ }^{1-3}$

Biliary carcinoma can be divided between gallbladder carcinoma and cholangiocarcinomas, since they have distinct biological behavior, probably because they have very different histological characteristics. Gallbladder carcinoma often mimics "pseudoklatskin" hilar cholangiocarcinomas. The rich submucosal layer of the gallbladder is connected to the large lymphatic chain of the hepatic and retroperitoneum wire, in addition to numerous cystic veins draining into the liver parenchyma. In this way, the carcinoma of the vesicle presents a more aggressive biological behavior, with distant metastatic potential. In contrast, cholangiocarcinomas present local and radial (superficial spread) infiltration and less frequent distant dissemination, probably due to their histology with scarce submucosal layer. ${ }^{4}$ 
Numerous potential postoperative problems may occur, in particular, hepatic insufficiency, which is still the main cause of failure of this procedure. Pancreatic fistulae occurring at high frequency are also prominent, since the caliber and consistency of the pancreatic gland are normal in these patients. Despite the recent advances in diagnostic methods and an increasing number of cases in early stages, most patients present advanced disease at the time of diagnosis, making R0 resections and curative surgeries difficult. However, the Japanese school demonstrated a significant improvement in the results of this operation through a rigorous preoperative preparation including routine portal embolizations for hypertrophy of the remaining hepatic lobe; systematic biliary drainage; endogenous bile replacement and thorough surgical technique. ${ }^{5}$
In Brazil, there is only one relevant publication about HPD in biliary cancer and some isolated groups in Rio Grande do Sul, São Paulo, Maranhão and Rio de Janeiro have gained experience in this operation. In three different institutions in Rio de Janeiro, the Hospital Adventista Silvestre, Hospital São Lucas and Hospital Universitario Clementino Fraga Filho-UFRJ, 35 patients underwent HPD between 2004 and 2014, representing, in our knowledge, the largest worldwide experience outside of Japan. ${ }^{6}$

The history of surgical treatment of biliary tract dates back $100 y e a r s$. The radical operations employed in the treatment of biliary cancer were born in Japan in the late 1970s, gaining popularity in various Japanese centers. ${ }^{7}$ Table 1 shows highlights of HPD surgery.

Table I History of the evolution of Hepatopancreatoduodenectomy

\begin{tabular}{|c|c|}
\hline Author & Evolution of Hepatopancreatoduodenectomy \\
\hline Glenn $^{6}$ & Liver resection + PD in I patient with gallbladder cancer \\
\hline $\mathrm{Nishi}^{7}$ & Hepatectomy (extended with unknown resection) + PD in I patient with gallbladder cancer \\
\hline Kasumi $^{8}$ & Extended right lobectomy + PD in I patient with gallbladder cancer \\
\hline Yokoyama & Right lobectomy (National Survey Conducted) + PD in I patient with gallbladder cancer \\
\hline Takasaki ${ }^{9}$ & Extended right lobectomy + PD in 5 patients with gallbladder cancer \\
\hline Shinohara"l & Extended right lobectomy + PD in I patient with gallbladder cancer (survived 4years and 6months) \\
\hline Sugiura & Extended right lobectomy + PD in 14 patients and extended right lobectomy + PD in 2 patients with gallbladder cancer \\
\hline Hanyu ${ }^{34}$ & HLPD (First clinical application in the world) \\
\hline Mizumoto' ${ }^{12}$ & HPD in $24 I$ patients, I 84 with gallbladder cancer and 57 with bile duct cancer (National Study) \\
\hline Starzl & Simultaneous liver and pancreas transplantation (Organ Cluster Transplantation) \\
\hline Mieles & Honey Organ Cluster Transplantation \\
\hline Hanyu $u^{30}$ & Transhepatic embolization of the preoperative common hepatic artery, allowing HLPD without reconstruction of the hepatic artery \\
\hline Nakamura $^{7}$ & $\begin{array}{l}\text { HPD in } 478 \text { patients (including } 24 \text { who received HLPD) with gallbladder and gallbladder cancer ( } 320 \text { with gallbladder cancer, I I I with bile } \\
\text { duct cancer and } 47 \text { with other diseases) }\end{array}$ \\
\hline Nimura ${ }^{23}$ & HPD in 24 patients with gallbladder cancer and carcinoma of the bile duct \\
\hline Nakamura 9 & HPD in 7 patients with gallbladder carcinoma \\
\hline Kawasaki $^{24}$ & Extended right lobectomy + PD after transhepatic portal percutaneous obliteration in 3 patients \\
\hline
\end{tabular}

$\mathrm{PD}=$ pancreatoduodenectomy

HLPD=Hepatoligamentopacreatoduodenectomy

HPD=Hepatopancreatoduodenectomy

In 1954, Glenn et al. ${ }^{6}$ performed hepatopancreatoduodenectomy in a patient who achieved 5-year survival after surgery. The first successful challenge of hepatopancreatoduodenectomy in biliary carcinoma (right hepatectomy) occurred in 1976, in Japan, by Kasumi et al. ${ }^{8}$ In 1980, Takasaki et al. ${ }^{9}$ reported 5 cases of HPD, 3 postoperative deaths and 2 patients survived 5 and 16 months, respectively. Despite the questionable outcome, this group produced a wealth of knowledge of the surgical treatment of biliary cancer in subsequent years and extensive experience was accumulated by this group in the radical resection of biliary carcinoma. ${ }^{10}$ In 1986 Shinohara et al. ${ }^{11}$ reported survival of 4years and 6months in a patient undergoing right lobectomy+pancreatoduodenectomy (PD).

Mizumoto et al. ${ }^{12}$ in 1990 and Nakamura et al. ${ }^{13}$ in 1992 reported that the number of simultaneous liver and pancreas resections doubled, from 241 to 478 , within 2years, demonstrating the high acceptance of this operation in Japan. Over the years efforts were made to prevent postoperative complications, particularly liver failure, highlighting preoperative portal embolization. ${ }^{14}$ In 1992, Nakamura et al. ${ }^{13}$ reported 478 cases of HPD in Japan, 320 of which were from gallbladder cancer, 111 from cholangiocarcinoma, and 47 from other causes. In the western world there are few HPD citations, ${ }^{15}$ with 22 cases in biliary cancer.

\section{Indications of HPD in biliary carcinoma}

Despite advances in the management of obstructive jaundice, biliary tract infections and increasing radiological technology, early detection of biliary neoplasms remains a challenge in clinical practice worldwide. In Brazil, the epidemiology of biliary cancer is still little known and radical surgeries are still poorly disseminated and restricted to a few groups. Most of the biliary tumors present with obstructive jaundice and many of these already with tumor invasion to the hepatoduodenal ligament, duodenum, liver, and advanced lymph node involvement. As previously described, biliary carcinoma can be divided into gallbladder cancer and cholangiocarcinomas of the 
extra hepatic biliary tract, the latter being a better prognostic tumor and often tumors with locoregional growth, which allows radical resection more frequently. ${ }^{16-18}$ In this way, HPD is the only operation with the possibility of R0 resection in some types of biliary cancer and should be used in selected cases of gallbladder cancer with possibility of lymphatic dissemination to the posterior pancreatoduodenal lymph nodes and eventually of the anterior pancreatoduodenals and, obviously, in the cases of direct tumor infiltration in the duodenal wall and pancreatic head. ${ }^{19-21}$

Gallbladder carcinoma presents many peculiarities, with $75 \%$ of the tumors located in the vesicular fund and $25 \%$ in the infundibulum..$^{22}$ Thus, due to the rich submucosal network of the infundibulum region, the tumors of this region are generally more aggressive, frequently present with advanced lymph node disease, hepatoduodenal ligament involvement and may mimic a hilar PseudoKlatskin cholangiocarcinoma. Tumors at the bottom of the gallbladder are more likely to be resected and their treatment should invariably comprise hepatic resection. The extent of the operation is still a matter of discussion in specialized centers but the degree of tumor infiltration into the liver parenchyma should always be taken into account, since tumors that invade more than $3 \mathrm{~cm}$ in the liver may have tumor cells in every right medial sector and left as venous branches of the vesicle flow into the intrahepatic portal branches of the IVb, V and VIII segments. ${ }^{23}$

The main indications of HPD or PD in patients with advanced bladder cancer are
i. Direct invasion of the pancreas, duodenum or both
ii. Signs of hepatoduodenal ligament invasion
iii. Lymph node infiltration of peripancreatic lymph nodes
iv. Chain lymph nodes 13 affected.

Discussion of PD indications is still very controversial (Figure 1). ${ }^{18,24,25}$ However, advanced gallbladder carcinoma is associated with a high incidence of metastases to the hepatoduodenal ligament lymph nodes, posterior pancreatoduodenal lymph nodes, and the common hepatic artery.

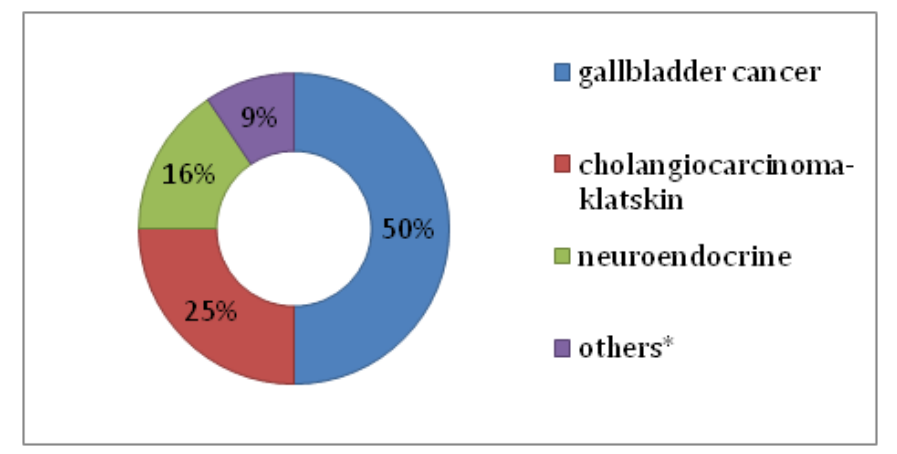

Figure I Major indications for HPD

There are basically 3 schools that differ on PD for resection of peripancreatic lymph nodes

a) PD is not indicated even in the presence of metastases, unless the metastases for lymph node infiltrate the pancreas

b) PD is indicated only if metastasis involvement is confirmed for posterior pancreatoduodenal lymph node

c) PD is indicated for regardless of the presence or absence of macroscopic lymph node metastases. ${ }^{21}$
Potential indications of HPD in cholangiocarcinoma

a) Diffuse tumor infiltration for any extrahepatic biliary tract

b) Perihilar tumor exhibiting superficial distal dissemination

c) Distal tumor exhibiting proximal and superficial dissemination

d) Tumor infiltrating the right hepatic artery and head of the pancreas

e) Perihilar tumor with lymph node metastasis bulky of the pancreatoduodenal region

f) Multiple tumors in the bile duct. Table 2 resumes total indications of HPD in our series.

Table 2 Mortality in HPD

\begin{tabular}{llll}
\hline Year & Autor & $\mathbf{N}^{\circ}$ Patients & Mortality \\
\hline 2001 & Yoshimi $^{31}$ & 13 & $7.70 \%$ \\
2007 & Miwa $^{28}$ & 26 & $0 \%$ \\
2007 & Kaneoka $^{27}$ & 20 & $15 \%$ \\
2008 & Wakai $^{29}$ & 28 & $21 \%$ \\
2010 & Hemming $^{30}$ & 22 & $0 \%$ \\
2012 & Ebata $^{26}$ & 79 & $2.40 \%$ \\
2013 & Sakamoto $^{32}$ & 19 & $5.30 \%$ \\
2014 & Fernandes $^{33}$ & $35(22$ Ca biliar) & $34.20 \%$ \\
\hline
\end{tabular}

\section{Methods}

We report a multicenter retrospective study, with 35patients, over 10years (2004-2014) in three different institutions in Rio de Janeiro, the Hospital Adventista Silvestre, Hospital São Lucas and Hospital Universitario Clementino Fraga Filho-UFRJ.

Surgery is initiated preferentially by the duodenopancreatic approach with extensive dissection of the hepatic wire and identification of possible vascular invasions. We do not recommend excessive manipulations of the vessels next to the tumor to guarantee R0 resections. Venous and arterial reconstructions are safer when performed while evacuating the surgical specimen.

A. First, the common or right common hepatic artery, which will feed the remaining liver in the future, is carefully dissected and preserved. The left portal vein is released from the hilar plaque allowing inspection of tumor invasion into the duct. As these steps are essential for $\mathrm{R} 0$ resection, the earliest possible preparation is recommended. The right hepatic artery and the gastroduodenal artery are ligated and sectioned. The stomach is sectioned $2 \mathrm{~cm}$ proximal of the pylorus when it is desired to preserve the pylorus or of the classic form transects the stomach to the level of the den. The superior mesenteric vein is exposed above and below the cervix of the pancreas.

B. Second, the jejunum is sectioned $10-20 \mathrm{~cm}$ distally to the Treitz angle. The first jejunal branch is taken in the play. The superior jejunum and the fourth portion of the duodenum pass through the surgical field on the right. The pancreatic nerve plexus between the uncinate process and the superior mesenteric artery is divided step by step along the lateral border of the superior mesenteric border. The extensive exposure of the mesenteric plateau and ligation of the pancreatoduodenal arteries in their origin with lymphadenectomy of this chain are mandatory. 
Then the cervix of the pancreas is divided and a drainage tube is placed in the wirsung after rigorous hemostasis. At this point the PD phase is complete.

C. Third, lymphadenectomy of the hepatoduodenal ligament is cephalad. The portal vein is repaired and dissected widely in the cranial direction. At this time, the hepatic lobe to be resected, more commonly the right one, and with that the ligature of the main portal branch assist in the ischemic demarcation of the hepatic parenchyma. The portal branches of the caudate lobe are carefully sectioned as well as the arantius canal in its origin on the left door. The mobilization ends with the section of the duct of arantius in its cephalic limit. Hepatic transection begins following the demarcation of the ischemic line. The pringle maneuver may be helpful in controlling bleeding. Hepatic transection can be performed in a variety of ways and with various energy and aspiration devices. However, kellyclasia can also be used safely. At the end of the transection, the piece surgery will only be attached to the hepatic vein of the wolf to be resected.

D. Finally, the pancreas, the intrahepatic bile duct and the duodenum are reconstructed in roux Y. Several pancreatic reconstructions can be used, including pancreatojejunostomy and pancreato-gastrostomy. Closure of the pancreatic stump can also be performed.

\section{Results}

In the 1990s, several Japanese centers published their initial results with HPD and there was an increase in indications for cholangiocarcinomas and a decreasing number of cases in the gallbladder carcinoma. In this way, more consistent results begin to be observed. Nimura et al. ${ }^{23}$ in 1991 reported 24 HPD with 6 deaths $(25 \%)$ especially related to postoperative liver failure and sepsis related to pancreatic fistula. Miyagawa et al. ${ }^{24}$ in 1996 demonstrated $0 \%$ mortality in HPD who underwent preoperative portal embolization and two-time pancreatic reconstruction. Table 3 shows the mortality rate worldwide.

Table 3 Indicationsof HPD

\begin{tabular}{|c|c|c|}
\hline Diagnosis & Indication & $\mathbf{n}$ \\
\hline \multirow{3}{*}{ Gallbladder cancer $(n=\mid 8)$} & $\begin{array}{l}\text { Peripancreatic lymph nodes } \\
\text { metastatis }\end{array}$ & 4 \\
\hline & Diffuse biliary infiltration & 4 \\
\hline & Duodenal/Pancreatic invasion & 10 \\
\hline \multirow{2}{*}{ Klatskin Tumor(n=8) } & Distal bile duct infiltration & 3 \\
\hline & Direct pancreatic invasion & 5 \\
\hline Neuroendocrine tumors $(n=5)$ & $\begin{array}{l}\text { Duodenal invasion from recurred } \\
\text { cancer }\end{array}$ & I \\
\hline \multirow{2}{*}{$\begin{array}{l}\text { Colorectal metastasis }(n=I) \\
\text { Metastatic intraductal papillary }\end{array}$} & $\begin{array}{l}\text { Liver metastasis and pancreatic } \\
\text { tumor }\end{array}$ & 4 \\
\hline & $\begin{array}{l}\text { Recure liver tumor with duodenal } \\
\text { invasion }\end{array}$ & 1 \\
\hline Mucinous neoplasm $(n=I)$ & Direct liver infiltration & 1 \\
\hline Metastatic melanoma $(\mathrm{n}=\mathrm{I})$ & Metastatic ocular melanoma & 1 \\
\hline Gastric cancer recurrence $(n=I)$ & $\begin{array}{l}\text { Tumor invasion to liver and } \\
\text { pancreatic head }\end{array}$ & 1 \\
\hline
\end{tabular}

Shirai et al. ${ }^{25}$ reported a 5 -year survival rate of $29 \%$ in patients with stage IVB gallbladder cancer undergoing HPD. The 5-year survival was $50 \%$ in R0 resection, in a group of 17 patients undergoing HPD. Of these, 5 patients were stage IVB and in them the survival was $29 \%$ and concludes that even with advanced disease there is benefit to the realization of HPD. $29 \%$ and conclude that even with advanced disease there is benefit to the performance of HPD. In the decade of 2000, numerous reports of HPD were reported with results superior to that of the previous decade and the experience accumulated in Japanese centers contributed to an important advance in the results of radical surgery of bile carcinoma.

Our group has pioneered the radical operations of biliary carcinoma with HPD in Brazil. However, the initial results were poor with high rates of pancreatic fistulas and hepatic failure with subsequent sepsis and death. The use of protocols from the accumulated experience of the Japanese school, such as the oral replacement of bile in jaundiced patients, routine portal embolization, immunonutrition and the greater use of centralectomies in gallbladder carcinomas instead of trisectionectomy have shown a significant improvement in the results. In the 35 cases evaluated, the overall mortality was $34.2 \%$ and in 17 patients $(53 \%)$ vascular resections and reconstruction were applied. Figure 2 shows types of hepatectomy employed and Figure 3 specimen's photos.

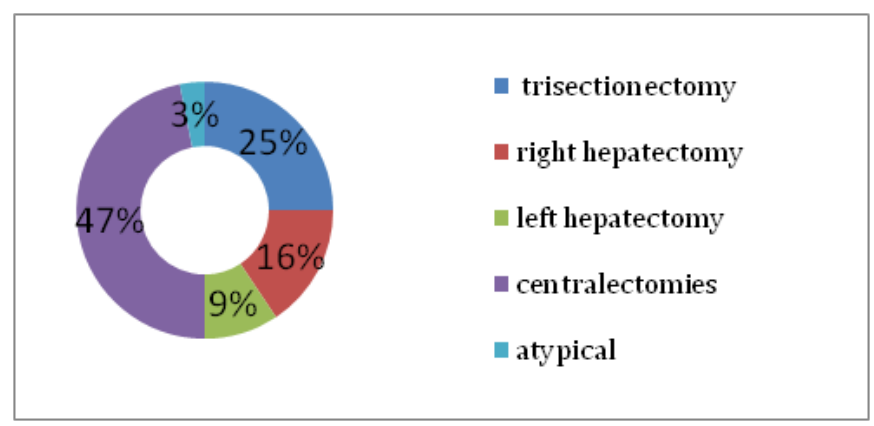

Figure 2 Types of Hepatectomies.

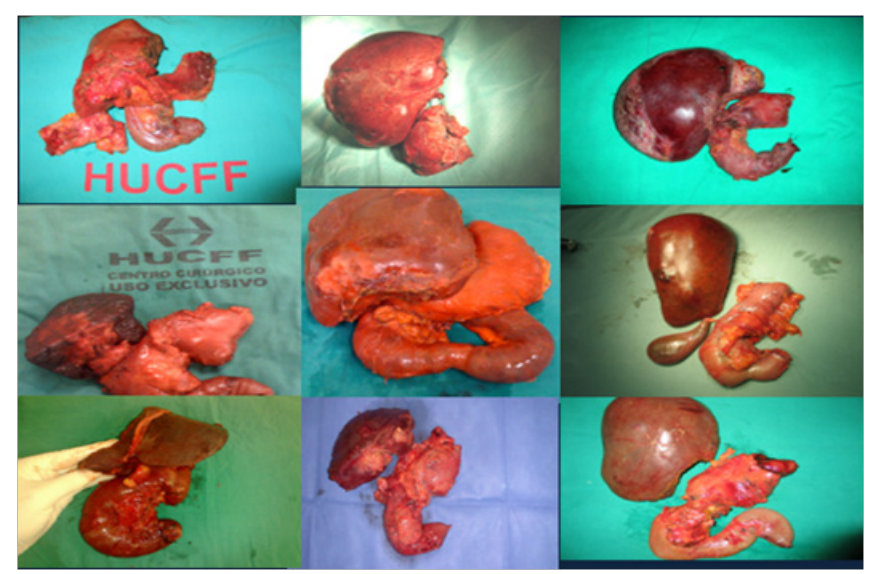

Figure 3 HPD cases.

\section{Discussion}

HPD was introduced in Japan in the 70's to treat advanced biliary carcinoma. ${ }^{14,15,21}$ Japanese specialized surgeons have developed significant experience in the surgical management of patients with advanced hepatopancreaticoduodenal cancer. ${ }^{1,15,19,22,24}$ In the western hemisphere, very few groups are interested in reproducing this experience. So far, only Hemming et al..$^{15}$ published a consisted 
report with HPD in the western countries. Nonetheless, a growing number of surgeons are engaged in developing more expertise with it in this decade. In the southern hemisphere, our groups have the largest HPD experience outside Japan. Ebata et al. ${ }^{3}$ define it as a multi-visceral resection combining a major liver resection and a pancreatoduodenectomy, which obtains an en-block specimen that, encircles the entire extra-hepatic biliary system. In this study, all types of major liver resections with pancreatoduodenectomy performed simultaneously, called HPD. ${ }^{7}$ In 1990's Japanese reports with it, including Nimura'set al. ${ }^{2}$ initial series with 24 cases and $25 \%$ mortality, mainly by liver failure and sepsis. Shirai et al. ${ }^{25}$ reported seven HPDs with 29\% mortality rate. Miyagawa et al. ${ }^{24}$ in 1996 published $0 \%$ mortality in seven cases and routine preoperative portal vein embolization. Shirai et al..$^{25}$ in 1997 reported 17 cases in stage IV gallbladder cancer and the 5-years survival was $29 \%$ and those with R0 resection the 5 -years survival was $50 \%$. The authors learned from this series that HPD is a very complex operation and the combination of right lobectomy or right trisectionectomy has a high mortality rate and such procedures requires a mandatory portal vein embolization to ensure a liver remnant large enough to avoid liver dysfunction. Likewise, preoperative biliary drainage and bile replacement are also important tools to guarantee better clinical status. The so called "Nagoya's protocol" appears to be instrumental in order to achieve lower postoperative mortality. ${ }^{18}$ The benefits of preoperative biliary drainage and bile re-infusion improving mitochondrial function, promoting adequate liver regeneration and also improving intestinal mucosa immunity to protect against bacterial translocation, has been well documented. ${ }^{2,12}$

Ebata et al. ${ }^{26}$ reported that hepatic failure rates decreased from $56 \%$ to $14 \%$ after routine preoperative portal vein embolization. In the 2000 's, because portal vein embolization has been widely used in the preoperative management, a lower incidence of liver failure was observed and many reports with zero mortality following HPD. Interestingly, many studies showed a worse survival in gallbladder cancer when compared to cholangiocarcinomas. All this cumulative knowledge has encouraged many eastern surgeons to perform HPD in cholangiocarcinomas with very good results. ${ }^{10}$ Ebata et al. ${ }^{26}$ reported a 10 -years survival of $32.1 \%$ with it for cholangiocarcinomas. Kaneoka et al. ${ }^{27}$ and Miwa et al. ${ }^{28}$ also reported excellent 5-years survival for HPD in cholangiocarcinomas $52 \%$ and $64 \%$, respectively. Wakai et al. ${ }^{29}$ in 2008 published 28 cases with perioperative mortality of $21 \%$. We acknowledge the weaknesses of this report where 11patients (31.4\%) were lost to follow-up. This is mainly related to the public health system in a very large country where patients need to travel long distances to get to specialized centers and many of those patients returned home were advanced medical care is scarce. Only 12 cases $(34.2 \%)$ had long term follow-up in this series. ${ }^{8}$ During 10 -years period, many improvements and experience were collected in the management of biliary carcinoma by the partners of this study. Using the Nagoya's team protocol refined our approach to HPD. All the complications observed in this period were the same observed by Japanese surgeons in the 90's. The combination of right trisectionectomy right lobectomy with pancreatoduodenectomy is feasible but mandatory portal vein embolization is required to obtain good postoperative survival because our results suggest that mortality over $40 \%$ is seen in those patients who underwent HPD (Group 1 and 2). So far, long term survival (9-years) was observed only in one patient who underwent right trisectionectomy plus pancreatoduodenectomy and portal vein reconstruction for gallbladder cancer and two patients with complex central hepatectomies plus pancreatoduodenectomy are also free of tumors recurrence in this series. Another four patients are alive and free of disease but the follow-up interval is short. ${ }^{10,30-33}$

\section{Conclusion}

HPD is a complex abdominal operation with a high mortality rate and a long learning curve. We emphasize that this operation should be limited to specialized centers with experience in liver transplantation background and advanced hepatobiliopancreatic surgery. Aggressive preoperative management with biliary drainage and portal vein embolization are crucial maneuvers to obtain satisfactory results. The HPDs for gallbladder cancer is feasible but requires a careful patient selection.

\section{Acknowledgements}

None.

\section{Conflicts of interest}

The authors declared that there are no conflicts of interest.

\section{References}

1. Nagino M, Ebata T, Yokoyama Y, et al. Evolution of Surgical Treatment of Perihilar Cholangiocarcinoma. Ann Surg. 2013;258(1):129-140.

2. Sakamoto E, Nimura Y, Hayakawa N, et al. The pattern of infiltration ate the proximal border of hilar bile duct carcinoma: a histologic analysis of 62 resected cases. Ann Surg. 1998;227(3):405-411.

3. Ebata T, Watanabe H, Ajioka Y, et al. Pathological appraisal of lines of resection for bile duct carcinoma. Br J Surg. 2002;89(10):1260-1267.

4. Igami T, Nagino $M$, Oda $K$, et al. Clinicopathologic Study of Cholangiocarcinoma with superficial spread. Ann Surg. 2009;249(2):296-302.

5. Ebata T, Kamiya J, Nishio H, et al. The concept of perihilarcholangiocarcinoma is valid. Br J Surg. 2009;96(8):926-934 .

6. Glenn F, Hays DM. The Scope of radical surgery in the treatment of malignant tumors of the extrahepatic biliary tract. Surg Gynecol Obstet. 1954;99(5):529-541.

7. Nishi M, Nemoto T, Misumi A, et al. Cancer of Gallbladder: A clinical and pathological study of 18 operative cases. Jpn J Cancer Clin . 1964;10:869-887.

8. Kasumi F, Tagagi K, Konishi T, et al. Surgical treatment of carcinoma of the gallbladder, analyzing its modes of spread. Nippon Shokaki Geka Gakkai Zasshi. 1976;9:170-177.

9. Takasaki K, Kobayashi S, Mutou H, et al. Our experiences (five cases) of extended hepatectomy for carcinoma of the gallbladder. Tan to Sui. 1980;7:923-932.

10. Tatsuo Araida, Tatsuya Yoshikawa, Tsukasa Azuma, Takeheiro Ota, Ken Takasaki, et al. (2004) Indications for pancreatoduodenectomy in patients undergoing lymphadenectomy for advanced gallbladder carcinoma. $J$ Hepatobiliary Pancreat Surg. 2004;11(1):45-49.

11. Shinohara T, Mizuno Y, Okada M, et al. A patient with advanced carcinoma of gallbladder surviving for 4years and 6months, following extended right hepatic lobectomy combined with pancreato-duodenectomy. Nippon Rinsho Geka Gakkai Zasshi. 1986;47:1656-1660.

12. Mizumoto R, Ogura Y, Matsuda S, et al. Cooperative study of surgical treatment of carcinoma of the biliary tract in Japan. Tan to Sui. 1990;11:869-882.

13. Nakamura M, Hanyu F, Araida T, et al. Pancreatoduodenectomy with hepatic resection. Tan to Sui. 1992;13:1305-1313. 
14. Makuuchi M, Thai BL, Takayasu K, et al. Preoperative portal embolization to increase safety of major hepatectomy foihilar bile duct carcinoma: a preliminary report. Surgery. 1990;107(5):521-527.

15. Hemming AW, Magliocca JF, Fujita S, et al. Combined resection of the liver and pancreas for malignancy. J Am Coll Surg. 2010;210(5):808-814.

16. Sugita M, Ryu M, Satake M, et al. Intrahepatic inflow areas of the drainage of the gallbladder : Analysis by angio- CT. Surgery. 2000;128(3):417-421.

17. Ebata T, Yokoyama Y, Igami T, et al. Hepatopancreatoduodenectomy for Cholangiocarcinoma. A Single center Review of 85 consecutive patients. Ann Surg. 2012;256(2):297-305.

18. Lim CS, Jang J, Lee S, et al. Reappraisal of hepatopancreatoduodenectomy as a treatment modality for bile duct and gallbladder cancer. $J$ GastrointestSurg. 2012;16(5):1012-1018.

19. Ebata T, Yokoyama Y, Igami $T$, et al. Review of hepatopancreatoduodenectomy for biliary cancer: an extended radical approach of Japanese origin. J Hepatobiliary Pancreat Sci. 2014;21(8):550-555.

20. Maplanta C. Gallbladder Cancer, Treatment Failure and Relapses: the peritoneum in Gallbladder Cancer. J Gastroint Canc 2014;45(3):245-255.

21. Nagino M, Ebata T, Yokoyama Y, et al. Evolution of Surgical Treatment for Perihilar Cholangiocarcinoma. Ann Surg. 2013;258(1):129-140.

22. Goldin R, Roa J. Gallbladder Cancer: A Morphological and molecular update. Histopathology. 2009;55(2):218-229.

23. Nimura Y, Hayakawa N, Kamiya J, et al. Hepatopancreatoduodenectomy for advanced carcinoma of the biliary tract. Hepatogastroenterology. 1991;38(2):170-175.
24. Miyagawa S, Makuuchi M, Kawasaki S, et al. Outcome of major hepatectomy with pancreatoduodenectomy for advanced biliary malignancies. World J Surg. 1996;20(1):77-80.

25. Shirai Y, Ohtani T, Tsukada K. Combined pancreatoduodenectomy and hepatectomy for patients with locally advanced gallbladder carcinoma: long term results. Cancer. 1997;80(10):2484-2492.

26. Ebata T, Yokayama Y, Igami T, et al. Hepatopancreatoduodenectomy for cholangiocarcinoma: a single center review of 85 consecutive patients. Ann Surg. 2012;256(2):297-305.

27. Kaneoka Y, Yamaguchi A, Isogai M. Hepatopancreatoduodenectomy: Is it suitable for bile duct cancer versus gallbladder cancer. J Hepatobiliary Pancret Surg. 2007;14(2):142-148.

28. Miwa S, Kobayashi A, Akahane Y, et al. Is major hepatectomy with pancreatoduodenectomy justified for advanced biliary malignancy? $J$ Hepatobiliary Pancreat Surg. 2007;14(2):136-141.

29. Wakai T, Shirai Y, Tsuchyia Y, et al. Combined major hepatectomy and pancreatoduodenectomy for locally advanced biliary carcinoma: long term results. World J Surg. 2008;32(6):1067-1074.

30. Yoshimi F, Asato Y, Amemiya R, et al. Comparison between pancreatoduodenectomy and hepatopancreatoduodenectomy for bile duct cancer. Hepatogastroenterology. 2001;48(40):994-998.

31. Sakamoto Y, Nara S, Kishi Y, et al. Is extended hemihepatectomy plus pancreatoduodenectomy justified for advanced bile duct cancer and gallbladder cancer? Surgery. 2013;153(6):794-800.

32. Fernandes E, Mello FT, Filho RJ, et al. The Largest western Experience with hepatopancreatoduodenectomy: Lessons learned with 35 cases. ABCD Arq Bras Cir Dig. 2016;29(1):17-20.

33. Hanyu F. Hepatopancreatoduodenectomy. Germany: Springer; 1996. 